\title{
Article
}

\section{SOMCL-085, a novel multi-targeted FGFR inhibitor, displays potent anticancer activity in FGFR-addicted human cancer models}

\author{
Xi-fei JIANG ${ }^{1, \#}$, Yang DAI ${ }^{2, \#}$, Xia PENG ${ }^{2}$, Yan-yan SHEN², Yi SU², Man-man WEI ${ }^{3}$, Wei-ren LIU $^{1}$, Zhen-bin DING ${ }^{1}$, Ao ZHANG ${ }^{2}$, \\ Ying-hong $\mathrm{SHI}^{1, *}$, Jing $\mathrm{Al}^{2,}$ * \\ ${ }^{1}$ Department of Liver Surgery, Liver Cancer Institute, Zhongshan Hospital, Fudan University, Key Laboratory of Carcinogenesis and \\ Cancer Invasion of Ministry of Education, Shanghai 200032, China; ${ }^{2}$ Division of Anti-Tumor Pharmacology, State Key Laboratory of \\ Drug Research, Shanghai Institute of Materia Medica, Chinese Academy of Sciences, Shanghai 201203, China; ${ }^{3}$ CAS Key Laboratory \\ of Receptor Research, and Synthetic Organic \& Medicinal Chemistry Laboratory (SOMCL), Shanghai Institute of Materia Medica, \\ Chinese Academy of Sciences, Shanghai 201203, China
}

\begin{abstract}
Aberrant fibroblast growth factor receptor (FGFR) activation is found across a diverse spectrum of malignancies, especially those lacking effective treatments. SOMCL-085 is a novel FGFR-dominant multi-target kinase inhibitor. Here, we explored the FGFRtargeting anticancer activity of SOMCL-085 both in vitro and in vivo. Among a panel of 20 tyrosine kinases Screened, SOMCL-085 potently inhibited FGFR1, FGFR2 and FGFR3 kinase activity, with $\mathrm{IC}_{50}$ values of 1.8, 1.9 and 6.9 nmol/L, respectively. This compound simultaneously inhibited the angiogenesis kinases VEGFR and PDGFR, but without obvious inhibitory effect on other 12 tyrosine kinases. In 3 representative human cancer cell lines with different mechanisms of FGFR activation tested, SOMCL-085 (20-500 $\mathrm{nmol} / \mathrm{L})$ dose-dependently inhibited FGFR1-3 phosphorylation and the phosphorylation of their key downstream effectors PLCy and Erk. In 7 FGFR aberrant human cancer cell lines, regardless of the mechanistic complexity of FGFR over-activation, SOMCL-085 potently inhibited FGFR-driven cell proliferation by arresting cells at the $\mathrm{G}_{1} / \mathrm{S}$ phase. In the FGFR1-amplified lung cancer cell line H1581 xenograft mice and FGFR2-amplified gastric cancer cell line SNU16 xenograft mice, oral administration of SOMCL-085 (25, 50 $\mathrm{mg} \cdot \mathrm{kg}^{-1} \cdot \mathrm{d}^{-1}$ ) for 21 days substantially suppressed tumor growth without affecting their body-weight. These results suggest that SOMCL085 is a potent multi-target FGFR inhibitor that inhibits the FGFR-dependent neoplastic phenotypes of human cancer cells in vitro and in vivo.
\end{abstract}

Keywords: human cancer; anticancer drug; SOMCL-085; receptor tyrosine kinase; FGFR; xenograft nude mouse model

Acta Pharmacologica Sinica (2018) 39: 243-250; doi: 10.1038/aps.2017.96; published online 14 Sep 2017

\section{Introduction}

The fibroblast growth factor receptor (FGFR) family of receptor tyrosine kinases (RTKs) comprises four members (FGFR1, FGFR2, FGFR3, and FGFR4) that share significant sequence homology. Receptor activation by FGFs initiates a cascade of intracellular events that activate major survival and proliferative signaling pathways mediating crucial physiological mechanisms, such as tissue and metabolic homeostasis, endocrine functions and wound repair ${ }^{[1-4]}$.

\footnotetext{
\# These authors contributed equally to this work.

* To whom correspondence should be addressed.

E-mail jai@simm.ac.cn (Jing Al);

shi.yinghong@zs-hospital.sh.cn (Ying-hong SHI)

Received 2017-05-24 Accepted 2017-06-20
}

Aside from their normal physiological roles described above, FGFs and FGFRs are emerging as oncogenes that drive proliferation in a significant proportion of human tumors and can also mediate resistance to both cytotoxic and targeted agents ${ }^{[1,4]}$. Deregulation of FGFR signaling has been documented in a broad spectrum of tumor types, in the form of FGFR gene amplifications, somatic mutations, or translocations, especially in tumors lacking effective treatment ${ }^{[1-4]}$. For example, amplification of FGFR1 (8q12locus) is found in approximately $17 \%$ of squamous nonsmall cell lung carcinoma (NSCLC) cases $^{[5,6]}$. Amplification of FGFR2 has been described in 5\%-10\% of gastric cancer cases, particularly of the aggressive diffuse subtype $e^{[7]}$, and in $4 \%$ of triple-negative breast cancer cases ${ }^{[8]}$. Mutations in FGFR3 are frequent in non-muscle invasive urothelial cell carcinomas and 
are also found in approximately $15 \%$ of high-grade invasive urothelial cancers $^{[9,10]}$. Thus, FGFR has been validated as an attractive target for cancer treatment.

Several FGFR inhibitors are undergoing clinical studies, but none of them have yet been approved for clinical use ${ }^{[4]}$. Many of the published FGFR inhibitors approved for VEGFR2based antiangiogenic therapies retain significant activity against VEGFR but have less potency against FGFRs ${ }^{[4,11-15]}$. Their activity against VEGFR is the likely source of grade $3 / 4$ hypertension induction and the dose-limiting toxicity of these inhibitors ${ }^{[16-19]}$. Most importantly, the angiogenesisregulating kinase VEGFR, PDGFR, and FGFR pathways are able to compensate for each other when single pathways are inhibited ${ }^{[20-23]}$. Accordingly, more recent treatments have focused on inhibiting multiple signaling pathways simultaneously.

Given the facts mentioned above, we sought to develop an orally bioavailable, FGFR-dominant kinase inhibitor that simultaneously inhibits VEGFR and PDGFR. To this end, we developed a novel triple kinase inhibitor of FGFR, VEGFR, and PDGFR, SOMCL-085. In the present study, we evaluated the FGFR-targeting antitumor activity of SOMCL-085 both in vitro and in vivo. The compound potently inhibited FGFR kinase activity and FGFR signal transduction, thereby suppressing the FGFR-dependent neoplastic phenotype of tumor cells. In xenografts of human lung and gastric tumor cell lines with FGFR-driven gene alterations, SOMCL-085 administration led to significant antitumor activity at well-tolerated doses. Our results suggest that SOMCL-085 has promising therapeutic potential for the treatment of FGFR-driven cancer in patients who acquire resistance to anti-VEGF/VEGFR2-based therapies.

\section{Materials and methods}

\section{Compound}

SOMCL-085 [6-((2-(4-(4-(2-hydroxyethyl)piperazin-1-yl)benzamido)pyridin-4-yl)oxy)- $N$-methyl-1-naphthamide hydrochloride] (Figure 1) was synthesized in Prof Ao ZHANG's Laboratory at the Shanghai Institute of Materia Medica. SOMCL-085 was dissolved in dimethyl sulfoxide at $10 \mathrm{mmol} / \mathrm{L}$ and subsequently serially diluted to specific concentrations.

\section{ELISA kinase assay}

The effects of SOMCL-085 on the activities of various tyrosine kinases were determined using enzyme-linked immunosorbent assays (ELISAs) with purified recombinant proteins. Briefly, $20 \mu \mathrm{g} / \mathrm{mL}$ poly (Glu, Tyr) 4:1 (Sigma, St Louis, MO, USA) was pre-coated in 96-well plates as a substrate. A $50 \mu \mathrm{L}$ aliquot of $10 \mu \mathrm{mol} / \mathrm{L}$ ATP solution diluted in kinase reaction buffer [ $50 \mathrm{mmol} / \mathrm{L}$ HEPES ( $\mathrm{pH} 7.4$ ), $50 \mathrm{mmol} / \mathrm{L} \mathrm{MgCl}_{2}, 0.5$ $\mathrm{mmol} / \mathrm{L} \mathrm{MnCl}_{2}, 0.2 \mathrm{mmol} / \mathrm{L} \mathrm{Na}_{3} \mathrm{VO}_{4}$, and $1 \mathrm{mmol} / \mathrm{L} \mathrm{DTT]}$ was added to each well; $1 \mu \mathrm{L}$ of the indicated compound diluted in $1 \%$ DMSO $(v / v)$ (Sigma, St Louis, MO, USA) was then added to each reaction well. DMSO $(1 \%, v / v)$ was used as the negative control. The kinase reaction was initiated by the addition of purified tyrosine kinase proteins diluted in

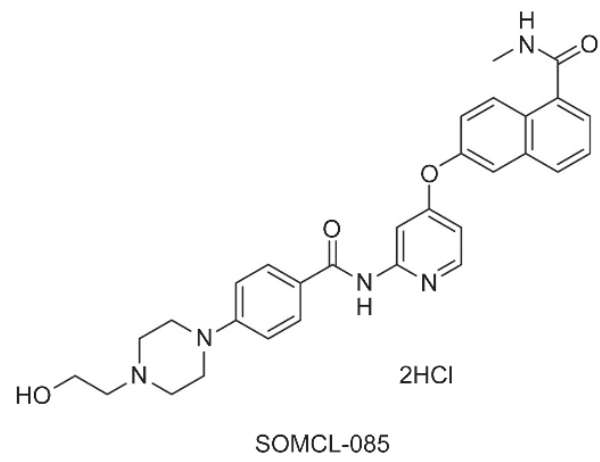

Figure 1. The chemical structure of SOMCL-085.

$49 \mu \mathrm{L}$ of kinase reaction buffer. After incubation for $60 \mathrm{~min}$ at $37^{\circ} \mathrm{C}$, the plate was washed three times with phosphatebuffered saline (PBS) containing $0.1 \%$ Tween 20 (T-PBS). Antiphosphotyrosine (PY99) antibody $(100 \mu \mathrm{L} ; 1: 500$, diluted in 5 $\mathrm{mg} / \mathrm{mL}$ BSA T-PBS) was then added. After a 30-min incubation at $37^{\circ} \mathrm{C}$, the plate was washed three times, and $100 \mu \mathrm{L}$ of horseradish peroxidase-conjugated goat anti-mouse IgG (1:1000, diluted in $5 \mathrm{mg} / \mathrm{mL}$ BSA T-PBS) was added. The plate was then incubated at $37^{\circ} \mathrm{C}$ for $30 \mathrm{~min}$ and washed three times. A $100-\mu \mathrm{L}$ aliquot of a solution containing $0.03 \% \mathrm{H}_{2} \mathrm{O}_{2}$ and $2 \mathrm{mg} / \mathrm{mL} o$-phenylenediamine in $0.1 \mathrm{~mol} / \mathrm{L}$ citrate buffer $(\mathrm{pH} 5.5)$ was added. The reaction was terminated by the addition of $50 \mu \mathrm{L}$ of $2 \mathrm{~mol} / \mathrm{L} \mathrm{H}_{2} \mathrm{SO}_{4}$ as the color changed, and the plate was analyzed using a multi-well spectrophotometer (SpectraMAX190, from Molecular Devices, Palo Alto, CA, USA) at $490 \mathrm{~nm}$. The inhibition rate (\%) was calculated using the following equation: $\left[1-\left(A_{490} / A_{490}\right.\right.$ control $\left.)\right] \times 100 \%$. The $\mathrm{IC}_{50}$ values were calculated from the inhibition curves in two separate experiments.

\section{Cell culture}

Human lung cancer cell line NCI-H1581, human acute myelogenous leukemia cell line KG1, human gastric cancer cell lines SNU16 and KATOIII, and human bladder cancer cell line NCIH716 were purchased from the American Type Culture Collection (ATCC, Manassas, VA, USA). Human bladder cancer cell line RT112 was purchased from the DSMZ-German collection of microorganisms and cell cultures. Human bladder cancer cell line UMUC14 was purchased from the European Collection of Cell Cultures (ECACC). All the cell lines were routinely maintained in media according to the suppliers' recommendations and authenticated via short tandem repeats analysis by Genesky Biopharma Technology (last tested in 2016).

\section{Western blot analysis}

KG1, SNU16 and UMUC14 cells were treated with the indicated dose of SOMCL-085 for $2 \mathrm{~h}$ at $37^{\circ} \mathrm{C}$ and then lysed in $1 \times$ SDS sample buffer. The cell lysates were subsequently resolved by 10\% SDS-PAGE and transferred to nitrocellulose membranes. The membranes were probed with the appropriate primary antibodies [phospho-FGFR, FGFR1, FGFR2, 
FGFR3, phospho-ERK, ERK, PLC $\gamma$, phospho-PLC $\gamma$, tubulin (all from Cell Signaling Technology, Beverly, MA, USA)], and then with horseradish peroxidase-conjugated anti-rabbit or anti-mouse IgG. The immune reactive proteins were detected using an enhanced chemiluminescence detection reagent (Thermo Fisher Scientific, Rockford, IL, USA).

\section{Cell proliferation assays}

Cells were seeded in 96-well plates at a low density in growth media. The next day, appropriate controls or designated concentrations of compounds were added to each well, and the cells were incubated for $72 \mathrm{~h}$. Finally, cell proliferation was determined using a sulforhodamine B (SRB) assay or a cell counting kit (CCK-8) assay. IC $_{50}$ values were calculated by concentration-response curve fitting using a SoftMax probased four-parameter method.

\section{Cell cycle analysis}

The effects of compounds on cell cycle progression and population distribution were determined by flow cytometry. Cells were seeded at $2 \times 10^{5}$ cells in 6 -well plates and treated with compounds at the indicated concentration or with vehicle as a control. After $24 \mathrm{~h}$, the cells were collected, fixed and stained with propidium iodide $(10 \mu \mathrm{g} / \mathrm{mL})$ for $30 \mathrm{~min}$ and then analyzed using a flow cytometer (FACSCalibur instrument; Becton, Dickinson \& Co, USA). Data were plotted using CellQuest software (Becton, Dickinson \& Co, USA).

\section{In vivo antitumor activity assay}

Female nude mice (4-6-weeks old) were housed and maintained under specific pathogen-free conditions. Animal procedures were performed according to the institutional ethical guidelines for animal care. Tumor cells at a density of $5 \times 10^{6}$ in $200 \mu \mathrm{L}$ were injected subcutaneously (sc) into the right flank of nude mice and then allowed to grow to $700-800 \mathrm{~mm}^{3}$, which was defined as a well-developed tumor. Subsequently, the well-developed tumors were cut into $1-\mathrm{mm}^{3}$ fragments and transplanted sc into the right flank of nude mice using a trocar. When the tumor volume reached $100-150 \mathrm{~mm}^{3}$, the mice were randomly assigned into either a vehicle control group $(n=12)$ or a treatment group ( $n=6$ per group). The control group was given only vehicle (water for injection), while the treatment groups received SOMCL-085 at the indicated doses via oral injection once daily for 2-3 weeks. The sizes of the tumors were measured twice per week using a microcaliper. Tumor volume $(\mathrm{TV})=\left(\right.$ length $\times$ width $\left.{ }^{2}\right) / 2$, and the individual relative tumor volume (RTV) was calculated as follows: RTV $=V_{\mathrm{t}} / V_{0}$, where $V_{\mathrm{t}}$ was the volume on a particular day and $V_{0}$ was the volume at the beginning of the treatment. The RTV was shown on indicated days as the median RTV \pm SE indicated for groups of mice. Percent (\%) inhibition (TGI) values were measured on the final day of study for the drug-treated mice compared with vehicle-treated mice and were calculated as $100 \times\left\{1-\left[\left(V_{\text {Treated Final day }}-V_{\text {Treated Day } 0}\right) /\left(V_{\text {Control Final day }}-V_{\text {Control Day 0 } 0}\right)\right]\right.$. Significant differences between the treated and control groups $(P \leq 0.05)$ were determined using the Student's $t$ test.

\section{Results}

SOMCL-085 is a potent FGFR-dominant kinase inhibitor that simultaneously inhibits VEGFR and PDGFR

SOMCL-085 (Figure 1) was selected for further characterization based on screens designed to identify a triple inhibitor of FGFR, VEGFR, and PDGFR. SOMCL-085 potently inhibited FGFR1-3 kinase activity, with $\mathrm{IC}_{50}$ values of $1.8,1.9$ and $6.9 \mathrm{nmol} / \mathrm{L}$, respectively (Figure 2, Table 1), and displayed weaker activity against FGFR4 $\left(\mathrm{IC}_{50}=319.9 \mathrm{nmol} / \mathrm{L}\right)$ (Figure 2, Table 1). Simultaneously, SOMCL-085 significantly inhibited VEGFR1, VEGFR2, PDGFR- $\alpha$, and PDGFR- $\beta$ kinase activity, with potency that was equal or lower than that observed against FGFR1-3. By contrast, no obvious inhibitory effect was observed against the other 12 tyrosine kinases tested from different families (Table 1). These data indicated that SOMCL-085 is a potent FGFR-dominant kinase inhibitor that simultaneously inhibits VEGFR and PDGFR. Here, we explored the in vitro and in vivo anti-FGFR activity of SOMCL085. The pharmacologic properties of SOMCL-085 in models associated with PDGFR and KDR will be described elsewhere.

\section{SOMCL-085 blocks FGFR activation and downstream signaling in cancer cells}

To further evaluate the cellular activity of SOMCL-085 targeting FGFR kinase, we analyzed its effects on the phosphorylation of FGFR and its major downstream signaling molecules, PLCY and ERK ${ }^{[1]}$. Three representative human cancer cell lines with different mechanisms of FGFR activation were used, including FGFR1-translocated myeloid leukemia cancer cell line KG-1, FGFR2-amplified gastric cancer cell

Table 1. The kinase panel screening data of SOMCL-085.

\begin{tabular}{|c|c|}
\hline Kinase & $\mathrm{IC}_{50}(\mathrm{nmol} / \mathrm{L})^{\mathrm{a}}$ \\
\hline FGFR1 & $1.8 \pm 0.2$ \\
\hline FGFR2 & $1.9 \pm 0.4$ \\
\hline FGFR3 & $6.9 \pm 1.2$ \\
\hline FGFR4 & $319.9 \pm 24.9$ \\
\hline VEGFR-1 & $5.6 \pm 0.5$ \\
\hline VEGFR-2 & $1.2 \pm 0.2$ \\
\hline PDGFR- $\alpha$ & $22.6 \pm 10.5$ \\
\hline PDGFR- $\beta$ & $7.8 \pm 1.8$ \\
\hline EGFR & $>1000$ \\
\hline ErbB2 & $>1000$ \\
\hline ErbB4 & $>1000$ \\
\hline $\mathrm{ABL}$ & $>1000$ \\
\hline c-Src & $>1000$ \\
\hline EPH-A2 & $>1000$ \\
\hline IGF1R & $>1000$ \\
\hline ALK & $>1000$ \\
\hline LTK & $>1000$ \\
\hline ROS1 & $>1000$ \\
\hline c-Met & $>1000$ \\
\hline$A X L$ & $>1000$ \\
\hline
\end{tabular}

${ }^{a}$ Values are the mean $\pm S D$ of two independent assays. 

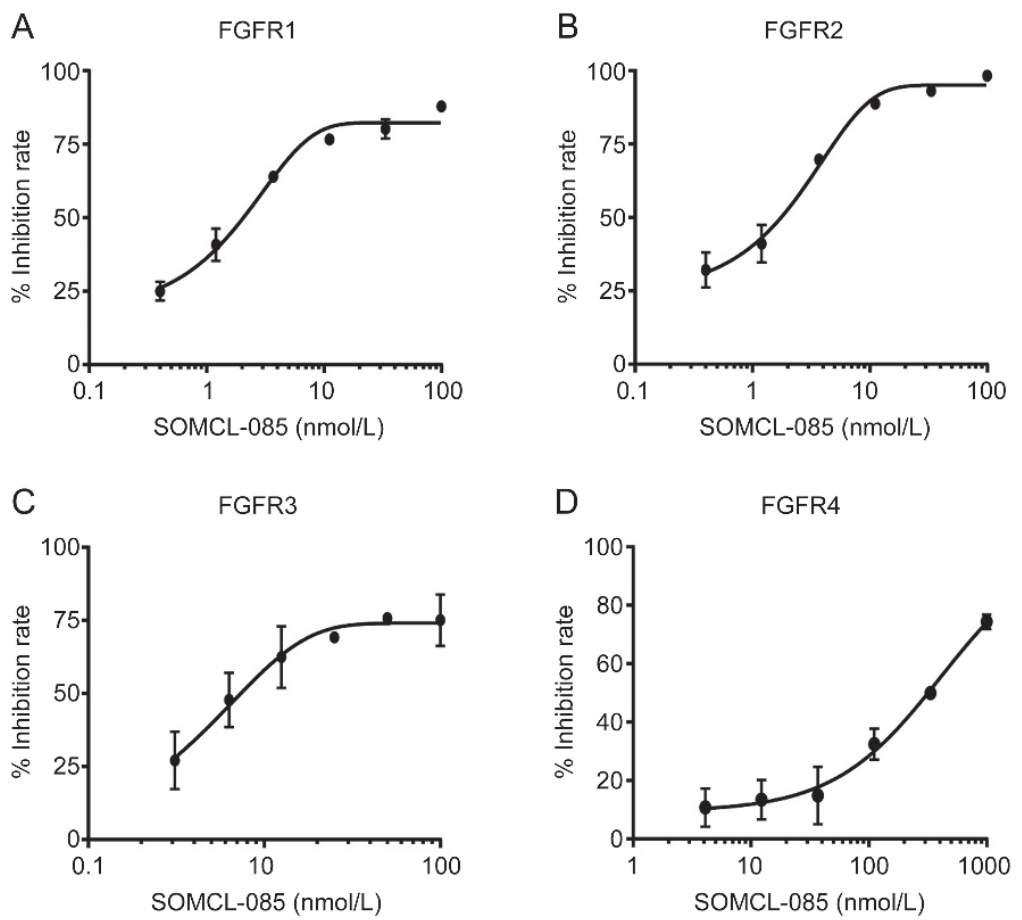

Figure 2. SOMCL-085 potently inhibited FGFR1, FGFR2, and FGFR3 kinase activity. The inhibition curve of SOMCL-085 against FGFR1 (A), FGFR2 (B), FGFR3 (C), and FGFR4 (D) kinase activity using ELISA assay.

line SNU16, and FGFR3-mutated bladder cancer cell line UMUC14. SOMCL-085 showed significant inhibition of FGFR1-3 phosphorylation in a dose-dependent manner in the individual cancer cell lines. The phosphorylation of ERK and PLCY was also clearly inhibited (Figure 3A-C). Thus, SOMCL-085 potently inhibits FGFR signaling, regardless of the mechanistic complexity of FGFR activation.

SOMCL-085 elicits significant effects against FGFR1-3-driven cancer cell proliferation via $\mathrm{G}_{1} / \mathrm{S}$ cell cycle arrest

To elucidate the impact of SOMCL-085 on FGFR-mediated cancer cell proliferation, seven cancer cell lines harboring the frequently occurring oncogenic forms of different FGFR members were chosen: FGFR1-translocated KG1 cells, FGFR1- amplified NCI-H1581 cells, FGFR2-amplified SNU16 cells, KATOIII cells, NCI-H716 cells, FGFR3-amplified RT112 cells, and FGFR3-mutated UMUC14 cells. The ability of SOMCL-085 to inhibit cell proliferation in these cells over a 3-day period was assessed using standard SRB and CCK8 proliferation assays. As shown in Figure 4A-G and Table S1, SOMCL-085 strongly inhibited FGFR1-, FGFR2-, and FGFR3driven cancer cell proliferation in a dose-dependent manner. The concentrations of SOMCL-085 required to inhibit cellular FGFR phosphorylation were in good agreement with the in vitro proliferation $\mathrm{IC}_{50}$ values (Table S1, Figure 3, Figure 4A$\mathrm{G})$, indicating that SOMCL-085 inhibited the tested cancer cell proliferation via targeted FGFR signaling.

FGFR inhibition is known to exert anti-proliferative effects
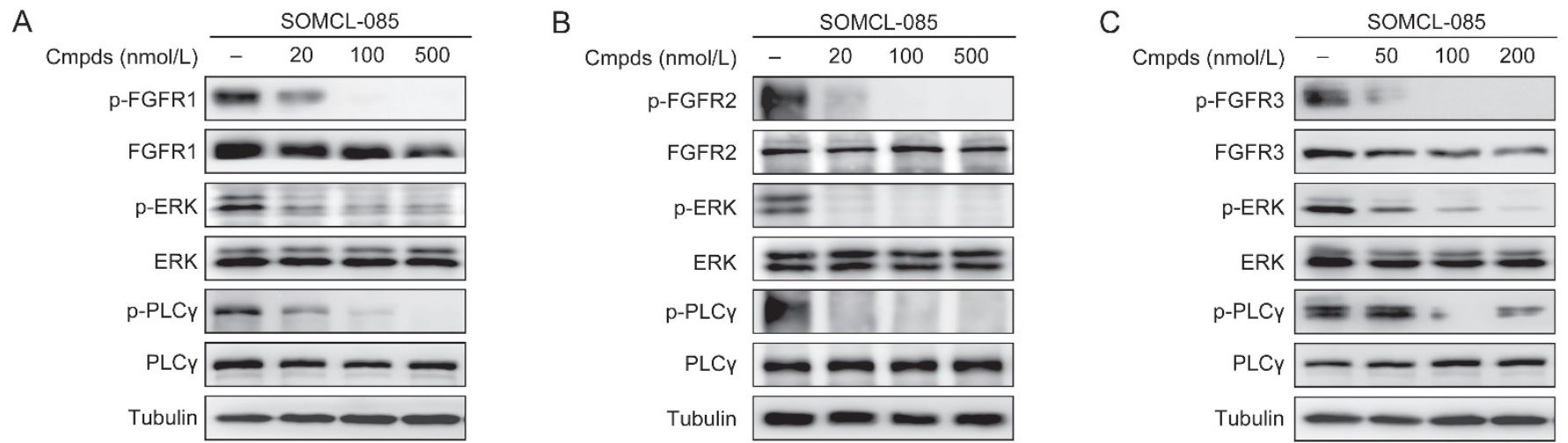

Figure 3. SOMCL-085 effectively inhibits the phosphorylation of FGFR and the downstream effectors Erk and PLCY in KG-1, SNU16, and UMUC14 cells. KG1 (A), SNU16 (B) or UMUC14 (C) cells treated with SOMCL-085 for $2 \mathrm{~h}$ at the indicated concentrations were lysed, and subjected to Western blot analysis. 
by arresting cells in the $\mathrm{G}_{1} / \mathrm{S}$ phase $\mathrm{e}^{[24]}$. To further confirm cellular FGFR targeting activity, we used a FGFR3-mutant UMUC14 cancer cell line as the representative FGFR-addicted context to measure cell-cycle distribution upon SOMCL-085 treatment. As expected, UMUC14 cells showed dramatic increases in the $G_{1}$ phase population upon treatment with SOMCL-085 (Figure 4H), with no increases in the sub-G population (data not shown), further confirming that the potent anti-proliferative activity of the compound in the FGFRaddicted context was the result of targeted FGFR signaling.

SOMCL-085 significantly inhibits FGFR-mediated tumor growth in vivo at well-tolerated doses

To assess the in vivo antitumor efficacy of SOMCL-085, the FGFR1-amplified lung cancer cell line NCI-H1581 and the FGFR2-amplified gastric cancer cell line SNU16 xenograft mouse models were used. In the SNU16 model, SOMCL-085 was orally administered once daily at doses of 25 or $50 \mathrm{mg} / \mathrm{kg}$ for 21 consecutive days. The results showed that SOMCL085 could suppress tumor growth in a dose-dependent manner, with tumor growth inhibition rates of $62.9 \%$ and $81.3 \%$ at doses of $25 \mathrm{mg} / \mathrm{kg}$ and $50 \mathrm{mg} / \mathrm{kg}$, respectively (Figure 5AC). Additionally, SOMCL-085 was well tolerated, with no significant body-weight loss in any of the treatment groups (Figure 5D). Similar results were observed in the NCI-H1581 model treated with SOMCL-085 (Figure 5E-H). These results indicated that SOMCL-085 elicited robust antitumor efficacy in FGFR-dependent tumor models at well-tolerated doses. Therefore, SOMCL-085 is a potential multi-target FGFR inhibitor for further drug development.

\section{Discussion}

Recent clinical success in molecular-targeted therapies for cancer based on the identification of oncogenic gene alterations
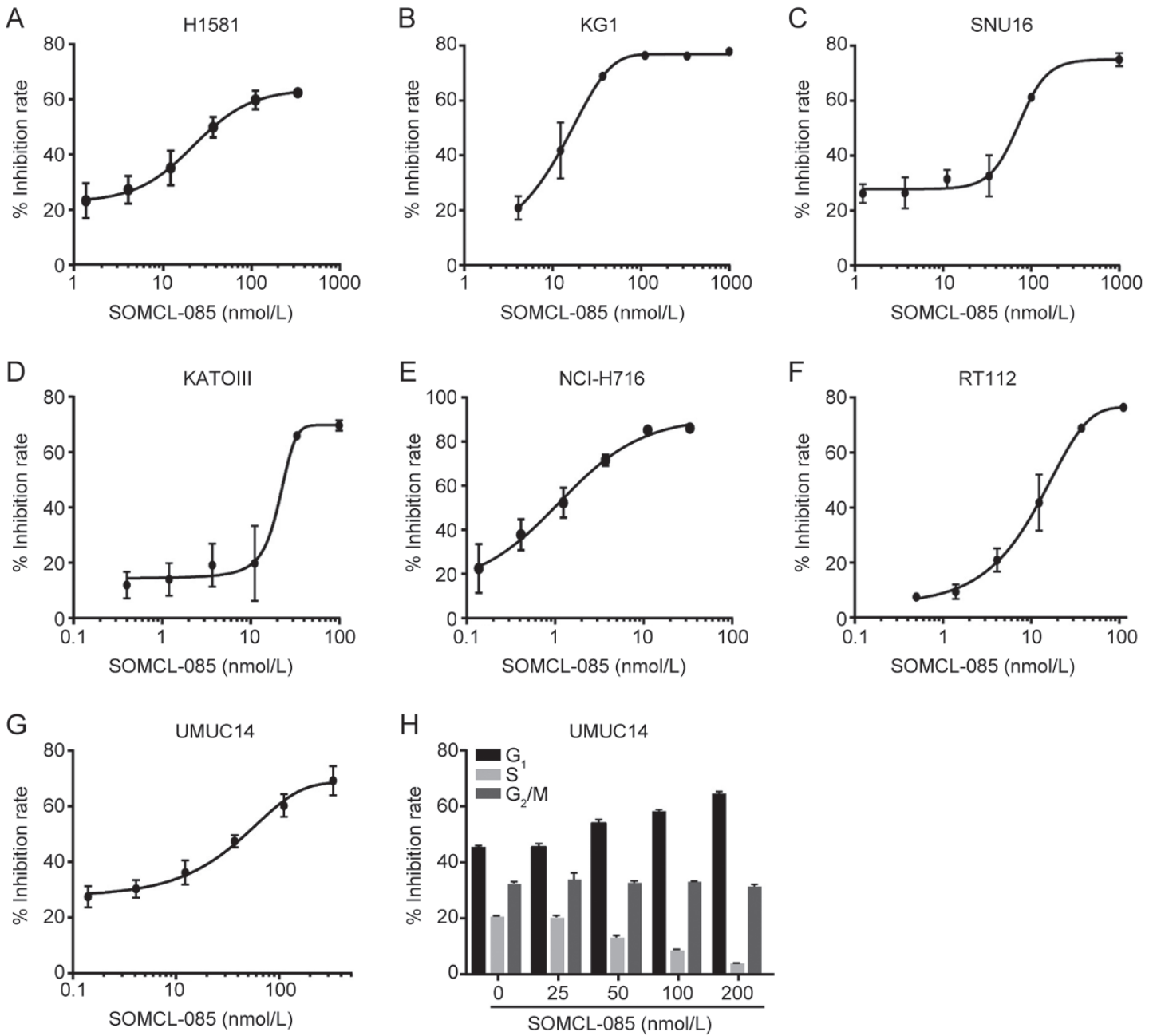

Figure 4. SOMCL-085 elicits significant effects against FGFR1-3-driven cancer cell proliferation via $\mathrm{G}_{1} / \mathrm{S}$ cell cycle arrest. (A-G) The anti-proliferative activity of SOMCL-085 against a panel of tumor cell lines, including NCI-H1581 (A), KG1 (B), SNU16 (C), KATOIII (D), NCl-H716 (E), RT112 (F), UMUC14 $(G)$, was determined by a sulforhodamine $B(S R B)$ assay or a CCK-8 assay. The inhibit rates were plotted as the mean \pm SD from two independent experiments. (H) SOMCL-085 induced $\mathrm{G}_{1} / \mathrm{S}$ phase cell cycle arrest in FGFR-addicted UMUC14 cancer cells. UMUC14 cells were treated with the indicated concentrations of SOMCL-085 for $24 \mathrm{~h}$. The percentages of cells in different cell cycle phases determined by FACS and analyzed with Modifit $\mathrm{LT}$ were plotted. The data shown are the mean $\pm \mathrm{SD}$ from three independent experiments. 

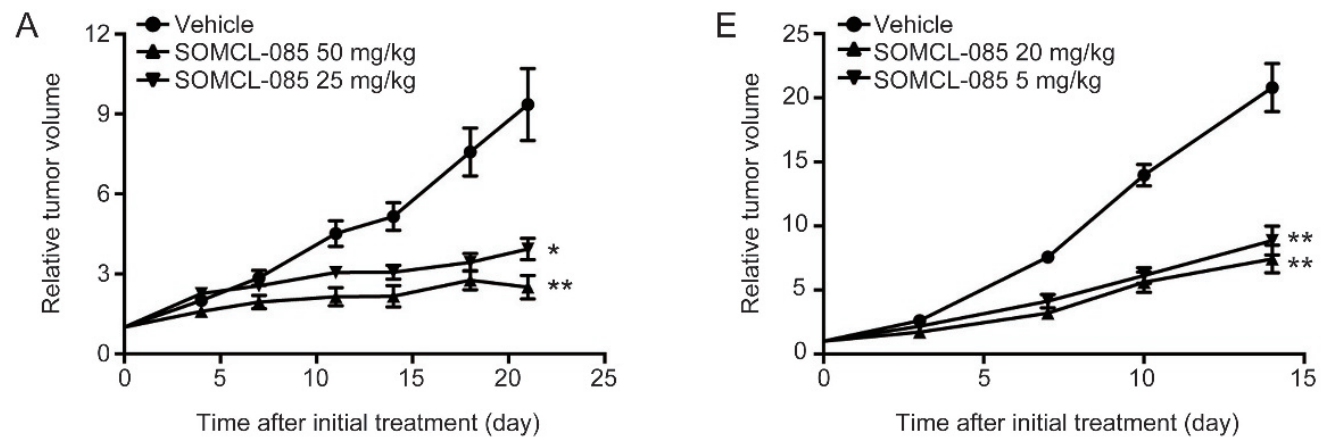

B

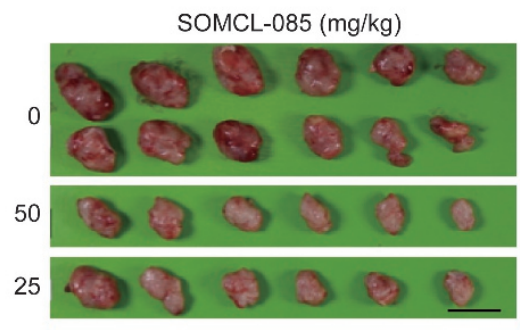

$\mathrm{F}$
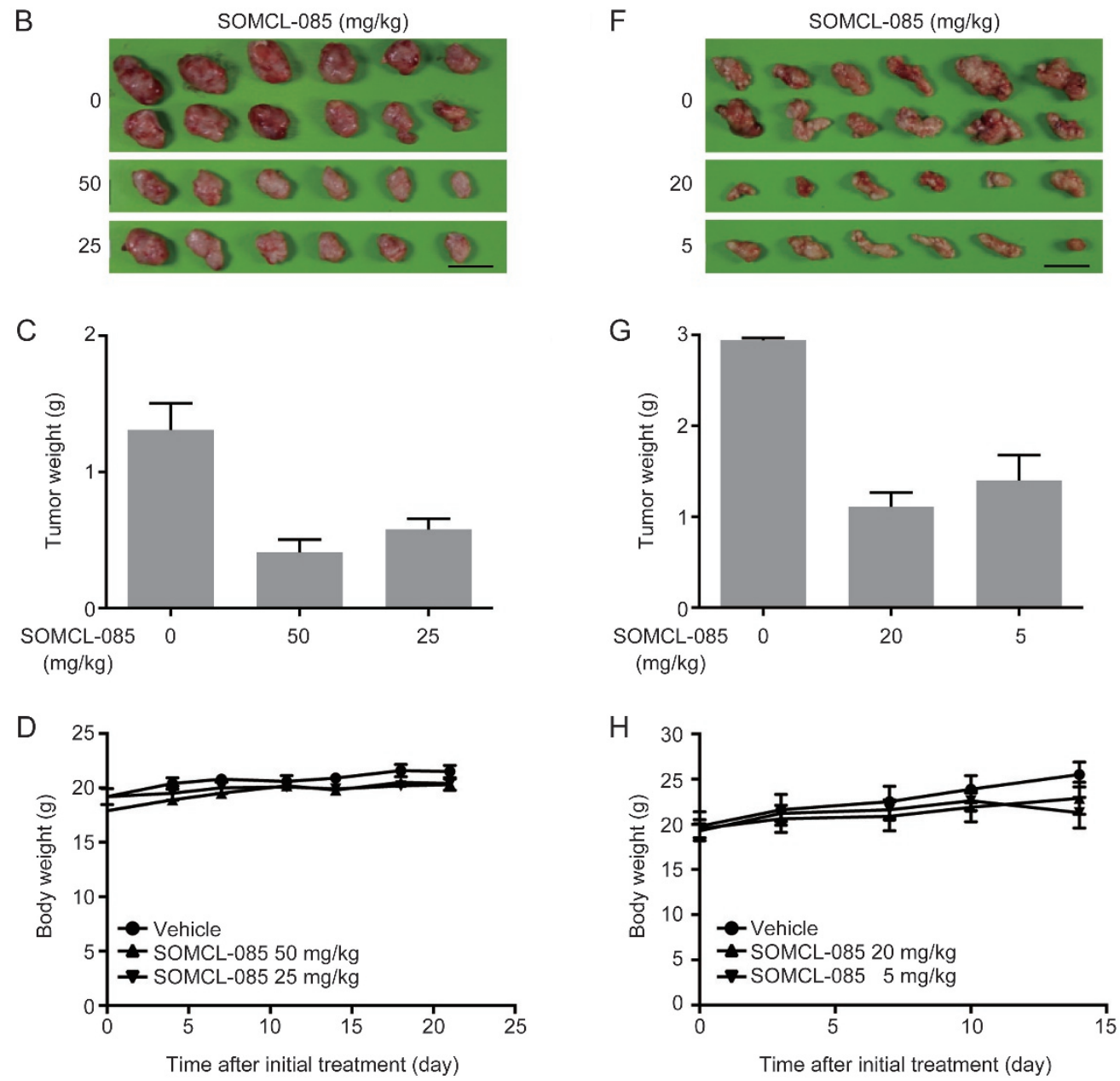

Figure 5. SOMCL-085 significantly inhibits FGFR-mediated tumor growth in vivo at well-tolerant doses. (A-D) Antitumor efficacy of SOMCL-085 in SNU16 xenograft model. (A) Tumor growth inhibition upon SOMCL-085 treatment in SNU16 xenografts was shown. The RTVs are expressed as the mean \pm SEM. Significant difference from the vehicle group was determined using a $t$-test, ${ }^{*} P<0.05,{ }^{* *} P<0.01$. Representative image of tumors (scale bar $=20$ $\mathrm{mm})(\mathrm{B})$ and tumor weights $(\mathrm{C})$ were shown on day 21 after mice injected with SOMCL-085. (D) Body weight measurements during the treatment. (E$\mathrm{H})$ Antitumor efficacy of SOMCL-085 in NCl-H1581 xenograft model. (E) Tumor growth inhibition upon SOMCL-085 treatment in NCl-H1581 xenografts was shown. The RTVs are expressed as the mean \pm SEM. Significant difference from the vehicle group was determined using a $t$-test, ${ }^{* *} P<0.01$. Representative image of tumors (scale bar=20 mm) $(\mathrm{F})$ and tumor weights $(\mathrm{G})$ were shown on day 14 after mice injected with SOMCL-085. (H) Body weight measurements during the treatment.

and their specific inhibitors has been associated with dramatic antitumor effects, reduced side effects, and improved patient survival. Mutant FGFR, a clinically relevant molecular oncogenic driver, is found across a diverse spectrum of malignancies, especially those lacking effective treatment ${ }^{[1,2,4]}$.
Aberrant FGFR activation is closely associated with tumor formation and metastasis, as well as resistance to approved therapies $^{[4,25-30]}$. Therefore, inhibiting FGFR signaling could have significant potential for the treatment of human cancers driven by FGFR over-activation. 
Here, we report the activity of the multi-targeted kinase inhibitor, SOMCL-085, against the FGFR family of kinases. Using FGFR-dependent cancer cells with different mechanisms of activation, we showed that SOMCL-085 potently inhibits the activation of FGFR1-3 signaling in cells and significantly inhibits cancer cell proliferation. Consistent with anti-proliferative effects mediated by inhibition of individual FGFRs, phosphorylation of the receptor was inhibited in all three cell lines with similar potency. In addition, using FGFR1-amplified H1581 and FGFR2-amplified SNU16 mouse xenograft models, we showed that daily oral administration of SOMCL-085 led to substantial inhibition of tumor growth at well-tolerated doses. Our study supports SOMCL-085 as a potent FGFR inhibitor that inhibits the activity of FGFRs regardless of their mechanism of activation.

It cannot be ignored that, as a FGFR-targeted inhibitor, SOMCL-085 simultaneously inhibits the angiogenesis kinases VEGFR and PDGFR. Although antiangiogenic therapies have proven to be effective in clinical settings, they also have well-characterized serious side effects that limit their clinical application. Most importantly, angiogenic kinase pathways including VEGFR, PDGFR, and FGFR can easily compensate for each other during blockade of a single pathway ${ }^{[20-23,30]}$. All of these findings suggest that a multi-targeting strategy towards both FGFR and VEGFR with PDGFR would provide a better treatment opportunity for patients who have disease progression following anti-VEGF/VEGFR2-based therapies. SOMCL-085 is an FGFR-dominant inhibitor with simultaneous inhibitory effects on VEGFR and PDGFR that may have promising treatment potential for patients who acquire resistance to anti-VEGF/VEGFR2-based therapies.

In summary, SOMCL-085 is a multi-targeted kinase inhibitor that displays potent pan-FGFR activity and inhibits the growth of cancer cells containing FGFR activated by multiple mechanisms. Several cancer indications contain genomic aberrations in FGFRs, and patients with these diseases tend to lack effective targeted therapies. These data strongly support the investigation of SOMCL-085 as a therapy for patients with FGFR-driven cancers

\section{Acknowledgements}

This work was supported by funds from the National Natural Science Foundation of China (№ 81473243), grants from the Science and Technology Commission of Shanghai Municipality (No 17431902900) and grants from the "Personalized Medicines-Molecular Signature-based Drug Discovery and Development", Strategic Priority Research Program of the Chinese Academy of Sciences (№ XDA12020000).

\section{Author contribution}

Jing AI and Ying-hong SHI designed the study; Xi-fei JIANG, Yang DAI, Yan-yan SHEN, Yi SU, Xia PENG, Wei-ren LIU, and Zhen-bin DING performed the research; Man-man WEI and Ao ZHANG contributed new reagents or analytic tools; Xi-fei JIANG, Xia PENG, and Jing AI analyzed the data; Xi-fei
JIANG, Xia PENG, and Jing AI wrote the paper.

\section{Supplementary information}

Supplementary information is available at the website of Acta Pharmacologica Sinica.

\section{References}

1 Turner N, Grose R. Fibroblast growth factor signalling: from development to cancer. Nat Rev Cancer 2010; 10: 116-29.

2 Dieci MV, Arnedos M, Andre F, Soria JC. Fibroblast growth factor receptor inhibitors as a cancer treatment: from a biologic rationale to medical perspectives. Cancer Discov 2013; 3: 264-79.

3 Touat M, lleana E, Postel-Vinay S, Andre F, Soria JC. Targeting FGFR Signaling in Cancer. Clin Cancer Res 2015; 21: 2684-94.

4 Babina IS, Turner NC. Advances and challenges in targeting FGFR signalling in cancer. Nat Rev Cancer 2017; 17: 318-32.

5 Yang W, Yao YW, Zeng JL, Liang WJ, Wang L, Bai CQ, et al. Prognostic value of FGFR1 gene copy number in patients with non-small cell lung cancer: a meta-analysis. J Thoracic Dis 2014; 6: 803-9.

6 Weiss J, Sos ML, Seidel D, Peifer M, Zander T, Heuckmann JM, et al. Frequent and focal FGFR1 amplification associates with therapeutically tractable FGFR1 dependency in squamous cell lung cancer. Sci Translation Med 2010; 2: 62ra93.

7 Matsumoto K, Arao T, Hamaguchi T, Shimada Y, Kato K, Oda I, et al. FGFR2 gene amplification and clinicopathological features in gastric cancer. Br J Cancer 2012; 106: 727-32.

8 Turner N, Lambros MB, Horlings HM, Pearson A, Sharpe R, Natrajan $\mathrm{R}$, et al. Integrative molecular profiling of triple negative breast cancers identifies amplicon drivers and potential therapeutic targets. Oncogene 2010; 29: 2013-23.

9 Helsten T, Elkin S, Arthur E, Tomson BN, Carter J, Kurzrock R. The FGFR Landscape in cancer: analysis of 4,853 tumors by nextgeneration sequencing. Clin Cancer Res 2016; 22: 259-67.

10 Cappellen D, De Oliveira C, Ricol D, de Medina S, Bourdin J, SastreGarau X, et al. Frequent activating mutations of FGFR3 in human bladder and cervix carcinomas. Nat Genet 1999; 23: 18-20.

11 Roth GJ, Heckel A, Colbatzky F, Handschuh S, Kley J, LehmannLintz $T$, et al. Design, synthesis, and evaluation of indolinones as triple angiokinase inhibitors and the discovery of a highly specific 6-methoxycarbonyl-substituted indolinone (BIBF 1120). J Med Chem 2009; 52: 4466-80.

12 Bhide RS, Cai ZW, Zhang YZ, Qian L, Wei D, Barbosa S, et al. Discovery and preclinical studies of $(R)$-1-(4-(4-fluoro-2-methyl- $1 \mathrm{H}$ indol-5-yloxy)-5- methylpyrrolo[2,1-f][1,2,4]triazin-6-yloxy)propan- 2-ol (BMS-540215), an in vivo active potent VEGFR-2 inhibitor. J Med Chem 2006; 49: 2143-6.

13 Wedge SR, Kendrew J, Hennequin LF, Valentine PJ, Barry ST, Brave SR, et al. AZD2171: a highly potent, orally bioavailable, vascular endothelial growth factor receptor-2 tyrosine kinase inhibitor for the treatment of cancer. Cancer Res 2005; 65: 4389-400.

14 Huynh H, Ngo VC, Fargnoli J, Ayers M, Soo KC, Koong HN, et al. Brivanib alaninate, a dual inhibitor of vascular endothelial growth factor receptor and fibroblast growth factor receptor tyrosine kinases, induces growth inhibition in mouse models of human hepatocellular carcinoma. Clin Cancer Res 2008; 14: 6146-53.

15 Sarker D, Molife R, Evans TR, Hardie M, Marriott C, ButzbergerZimmerli $\mathrm{P}$, et al. A phase I pharmacokinetic and pharmacodynamic study of TKI258, an oral, multitargeted receptor tyrosine kinase inhibitor in patients with advanced solid tumors. Clin Cancer Res 2008; 14: 2075-81. 
16 Herbst RS. Toxicities of antiangiogenic therapy in non-small-cell lung cancer. Clin Lung Cancer 2006; 8 Suppl 1: S23-30.

17 Izzedine H, Ederhy S, Goldwasser F, Soria JC, Milano G, Cohen A, et al. Management of hypertension in angiogenesis inhibitor-treated patients. Ann Oncol 2009; 20: 807-15.

18 Ricciardi S, Tomao S, de Marinis F. Toxicity of targeted therapy in nonsmall-cell lung cancer management. Clin Lung Cancer 2009; 10 : 28-35.

19 Shojaei F, Ferrara N. Role of the microenvironment in tumor growth and in refractoriness/resistance to anti-angiogenic therapies. Drug Resistance Updates 2008; 11: 219-30.

20 Zhao Y, Adjei AA. Targeting angiogenesis in cancer therapy: moving beyond vascular endothelial growth factor. Oncologist 2015; 20 : 660-73.

21 Batchelor TT, Duda DG, di Tomaso E, Ancukiewicz M, Plotkin SR, Gerstner E, et al. Phase II study of cediranib, an oral pan-vascular endothelial growth factor receptor tyrosine kinase inhibitor, in patients with recurrent glioblastoma. J Clin Oncol 2010; 28: 2817-23.

22 Casanovas O, Hicklin DJ, Bergers G, Hanahan D. Drug resistance by evasion of antiangiogenic targeting of VEGF signaling in late-stage pancreatic islet tumors. Cancer Cell 2005; 8: 299-309.

23 Abdollahi A, Folkman J. Evading tumor evasion: current concepts and perspectives of anti-angiogenic cancer therapy. Drug Resistance Updates 2010; 13: 16-28.

24 Liu H, Ai J, Shen A, Chen Y, Wang X, Peng X, et al. c-Myc Alteration determines the therapeutic response to FGFR inhibitors. Clin Cancer Res 2017; 23: 974-84.

25 Fernanda Amary M, Ye H, Berisha F, Khatri B, Forbes G, Lehovsky K, et al. Fibroblastic growth factor receptor 1 amplification in osteosarcoma is associated with poor response to neo-adjuvant chemotherapy. Cancer Med 2014; 3: 980-7.

26 Ware KE, Marshall ME, Heasley LR, Marek L, Hinz TK, Hercule P, et al. Rapidly acquired resistance to EGFR tyrosine kinase inhibitors in NSCLC cell lines through de-repression of FGFR2 and FGFR3 expression. PLoS One 2010; 5: e14117.

27 Yadav V, Zhang X, Liu J, Estrem S, Li S, Gong XQ, et al. Reactivation of mitogen-activated protein kinase (MAPK) pathway by FGF receptor 3 (FGFR3)/Ras mediates resistance to vemurafenib in human B-RAF V600E mutant melanoma. J Biol Chem 2012; 287: 28087-98.

28 Manchado E, Weissmueller S, Morris JP, Chen CC, Wullenkord R, Lujambio A, et al. A combinatorial strategy for treating KRAS-mutant lung cancer. Nature 2016; 534: 647-51.

29 Kim B, Wang S, Lee JM, Jeong Y, Ahn T, Son DS, et al. Synthetic lethal screening reveals FGFR as one of the combinatorial targets to overcome resistance to Met-targeted therapy. Oncogene 2015; 34: 1083-93.

30 Terai H, Soejima K, Yasuda H, Nakayama S, Hamamoto J, Arai D, et al. Activation of the FGF2-FGFR1 autocrine pathway: a novel mechanism of acquired resistance to gefitinib in NSCLC. Mol Cancer Res 2013; 11: 759-67. 